\title{
Role of Circulating miRNAs as Biomarkers in Idiopathic Pulmonary Arterial Hypertension: Possible Relevance of miR-23a
}

\author{
Irene Sarrion, ${ }^{1}$ Lara Milian, ${ }^{2,3}$ G. Juan, ${ }^{4,5}$ Mercedes Ramon, ${ }^{4,5}$ Idelfonso Furest, ${ }^{6}$ \\ Carmen Carda, ${ }^{2,7}$ Julio Cortijo Gimeno, ${ }^{8,9,10}$ and Manuel Mata Roig ${ }^{2,7,8}$ \\ ${ }^{1}$ Universidad Catolica de Valencia, Guillem de Castro Street 106, 46003 Valencia, Spain \\ ${ }^{2}$ Department of Pathology, Faculty of Medicine and Odontology, University of Valencia, Blasco Ibáñez Avenue 15, \\ 46010 Valencia, Spain \\ ${ }^{3}$ Fundación para el Fomento de la Investigación Sanitaria y Biomédica de la Comunitat Valenciana (FISABIO), \\ Cataluña Avenue 21, 46020 Valencia, Spain \\ ${ }^{4}$ Hospital General Universitario de Valencia, Tres Cruces Avenue, 46014 Valencia, Spain \\ ${ }^{5}$ Department of Medicine, Faculty of Medicine and Odontology, University of Valencia, Blasco Ibáñez Avenue 15, \\ 46010 Valencia, Spain \\ ${ }^{6}$ Hospital Universitario Doctor Peset, Gaspar Aguilar Street 90, 46017 Valencia, Spain \\ ${ }^{7}$ Fundación para la Investigación del Hospital Clínico de la Comunidad Valenciana (INCLIVA), Blasco Ibañez Avenue 15, \\ 46010 Valencia, Spain \\ ${ }^{8}$ Centro de Investigación Biomédica en Red de Enfermedades Respiratorias (CIBERES), Carretera Soller Km 12, Bunyola, \\ Mallorca, 07110 Illes Balears, Spain \\ ${ }^{9}$ Department of Pharmacology, Faculty of Medicine and Odontology, University of Valencia, Blasco Ibáñez Avenue 15, \\ 46010 Valencia, Spain \\ ${ }^{10}$ Fundación de Investigación Hospital General Universitario de Valencia, Tres Cruces Avenue 3, 46014 Valencia, Spain
}

Correspondence should be addressed to Manuel Mata Roig; mata_manroi@gva.es

Received 27 October 2014; Revised 1 February 2015; Accepted 4 February 2015

Academic Editor: Peter Backx

Copyright (C) 2015 Irene Sarrion et al. This is an open access article distributed under the Creative Commons Attribution License, which permits unrestricted use, distribution, and reproduction in any medium, provided the original work is properly cited.

Idiopathic pulmonary hypertension (IPAH) is a rare disease characterized by a progressive increase in pulmonary vascular resistance leading to heart failure. MicroRNAs (miRNAs) are small noncoding RNAs that control the expression of genes, including some involved in the progression of IPAH, as studied in animals and lung tissue. These molecules circulate freely in the blood and their expression is associated with the progression of different vascular pathologies. Here, we studied the expression profile of circulating miRNAs in 12 well-characterized IPAH patients using microarrays. We found significant changes in 61 miRNAs, of which the expression of miR23a was correlated with the patients' pulmonary function. We also studied the expression profile of circulating messenger RNA (mRNAs) and found that miR23a controlled $17 \%$ of the significantly changed mRNA, including PGCl $\alpha$, which was recently associated with the progression of IPAH. Finally we found that silencing of miR23a resulted in an increase of the expression of PGC1 $\alpha$, as well as in its well-known regulated genes CYC, SOD, NRF2, and HO1. The results point to the utility of circulating miRNA expression as a biomarker of disease progression.

\section{Introduction}

Pulmonary arterial hypertension (PAH) is a disease characterized by a progressive increase in pulmonary vascular resistance, causing an elevation in pulmonary artery blood pressure leading to fatal right heart failure $[1,2]$. PAH can be grouped into five categories according to etiology. Idiopathic PAH (IPAH) belongs to group 1 and includes patients with a mean pulmonary artery pressure (MPAP) $\geq$ $25 \mathrm{mmHg}$, a pulmonary capillary wedge pressure (PCWP), 
left atrial pressure, or left ventricular end-diastolic pressure $\leq$ $15 \mathrm{mmHg}$, and a pulmonary vascular resistance (PVR) greater than three Wood units [3]. IPAH is a rare disease, with a prevalence of 2-3 per million per year, in which reactive oxygen species- (ROS-) mediated events are important [4, 5]. Recently, the expression of the peroxisome proliferationactivated receptor (PPAR) $\gamma$ coactivator- $1 \alpha$ (PGC1 $\alpha$ ) has been proposed as a potential biomarker of the progression of IPAH [6]. This protein is involved in the control of the expression of PPAR-regulated genes involved in oxidative metabolism and mitochondrial biogenesis [7].

MicroRNAs (miRNAs) are small $(\approx 22$ nucleotides in length) endogenously expressed noncoding RNAs that regulate gene expression at the posttranscriptional level, inhibiting or degrading their target RNAs $[8,9]$. These molecules circulate freely in mammalian blood, and several studies have proposed that they can serve as biomarkers for different vascular pathologies, including early myocardial infarction and heart failure in humans [10-13]. miRNAs have been also implicated in the pathophysiological mechanisms involved in the progression of IPAH, including endothelial dysfunction, plexiform lesion formation, smooth muscle cell proliferation, and fibroblast activation and proliferation [14].

Recently, evidence has demonstrated the potential role of circulating miRNAs as biomarkers of the progression of the disease [15]. These studies have examined animal models or isolated lung tissue; to our knowledge, no specific global circulating miRNA expression studies have compared IPAH patients with healthy volunteers.

Therefore, this study used high-density microarrays to study circulating miRNAs in 12 well-characterized IPAH patients. Our results support the potential roles of different miRNAs including the miR23a, miR-130, miR-191, miR-204, miR-145, miR-27a, miR-328, miR-1-2, miR-199, and miR744 as biomarkers of IPAH. We also highlight the potential relevance of miR-23a because it correlates with the patient's pulmonary function as well as with the potential control of the expression of several circulating mRNAs in which PGCl $\alpha$ is included.

\section{Methods}

2.1. Study Subjects. This study included 12 well-characterized IPAH patients and ten healthy volunteers. The patient inclusion criteria were MPAP $>25 \mathrm{mmHg}$, PCWP $\leq 15 \mathrm{mmHg}$, and PVR > 3 Wood units measured by catheterization. All patients had been treated with different combinations of bosentan, treprostinil, nifedipine, and iloprost before sample collection. The detailed clinical features of the patients, as well as the superoxide dismutase (SOD), PGCl $\alpha$, cytochrome C (CYTC), total antioxidant status (TAS), and glutathione peroxidase (GPX) levels, have been reported [6] and are summarized in Table 1.

All experiments were approved by the Ethics Committee of the University General Hospital of Valencia (Spain) and informed consent was obtained. $5 \mathrm{~mL}$ of peripheral blood was extracted from each patient. $2.5 \mathrm{~mL}$ of peripheral blood was collected in PAXgene RNA collection tubes (QIAGEN, CA, USA) for total RNA extraction while the serum of the remaining $2.5 \mathrm{~mL}$ was reserved for miRNA extraction. Collected samples were stored at $-80^{\circ} \mathrm{C}$ until extraction.

2.2. Microarray Analysis. For miRNA global expression analysis, Affymetrix miRNA 2.0 arrays (Affymetrix, CA, USA) were used. miRNA was extracted from serum of the IPAH and healthy volunteers using the miRNeasy Serum/Plasma Kit as recommended by the manufacturer (QIAGEN, CA, USA). Extractions were biotin labeled using the 3DNA Array Detection FlashTag Biotin HSR (Genisphere, Hatfield, USA) and hybridized with the microarrays in a hybridization oven 645 (Affymetrix, CA, USA), following standardized protocols supplied by Affymetrix. Subsequent staining, washing, and scanning of the microarrays were performed using a Fluidic Station 450 and confocal GeneChip scanner 3000 7G (Affymetrix, CA, USA).

Using the GeneChip Command Console Software (AGCC, Affymetrix, CA, USA), DAT and CEL files were acquired. Probe set summarization, background adjustment (using the BC-CG), and quantile normalization were performed using the miRNA QC tool software (Affymetrix, CA, USA). All arrays included in this study met the minimum quality criteria defined by Affymetrix. Extracted data were imported using the dCHIP analysis software (http://www .hsph.harvard.edu/cli/complab/dchip) [16] and statistical analysis was conducted as reported [17].

Global RNA analysis profiles were studied using Affymetrix human genome U133 plus 2.0 arrays (Affymetrix, CA, USA). Total RNA was extracted from the PAXgene RA collection tubes, as described by the manufacturer. Amplification, labeling, hybridization, staining, washing, and scanning of the microarrays followed standardized protocols, with manufacturer-recommended reagents and instruments. DAT and CEL files were acquired using the GeneChip Command Console Software (AGCC, Affymetrix, CA, USA) and the microarrays were analyzed using dCHIP. The invariant set method was used to normalize the data, as reported in $[16,17]$.

\subsection{Human Small Vascular Pulmonary Artery Endothelial} Cells (HSVPAEC) Culture and miR23a Inhibition. Human small vascular pulmonary artery endothelial cells (HSVPAEC) were isolated and cultured from small vessel of lung as previously described [18]. Segments of pulmonary artery (2-4 $\mathrm{mm}$ internal diameter) were dissected free from parenchyma lung tissue, cut longitudinally, and digested with $1 \%$ collagenase (Gibco, UK) in RPMI-1640 culture medium for $30 \mathrm{~min}$ at $37^{\circ} \mathrm{C}$. The digestion was neutralized by adding RPMI-1640 supplemented with $20 \%$ fetal calf serum (FCS), and the homogenate was separated by centrifugation at $1100 \mathrm{rpm}$. The pellet was resuspended, and the cells were cultured in EGM-2 endothelial culture medium supplemented with Single Quotes (Clonetics, UK), $10 \%$ FCS, $1 \%$ fungizone, and 2\% streptomycin/penicillin. HSVPAEC were selected using available Dynabeads CD31 endothelial cell kit (Dynal Biotech, Germany). The cells were trypsinized $(0.25 \%$ trypsin $)$ and incubated with CD31-coated Dynabeads for $30 \mathrm{~min}$ at $4^{\circ} \mathrm{C}$ with end-over-end rotation. After incubation, the HSVPAEC were collected 


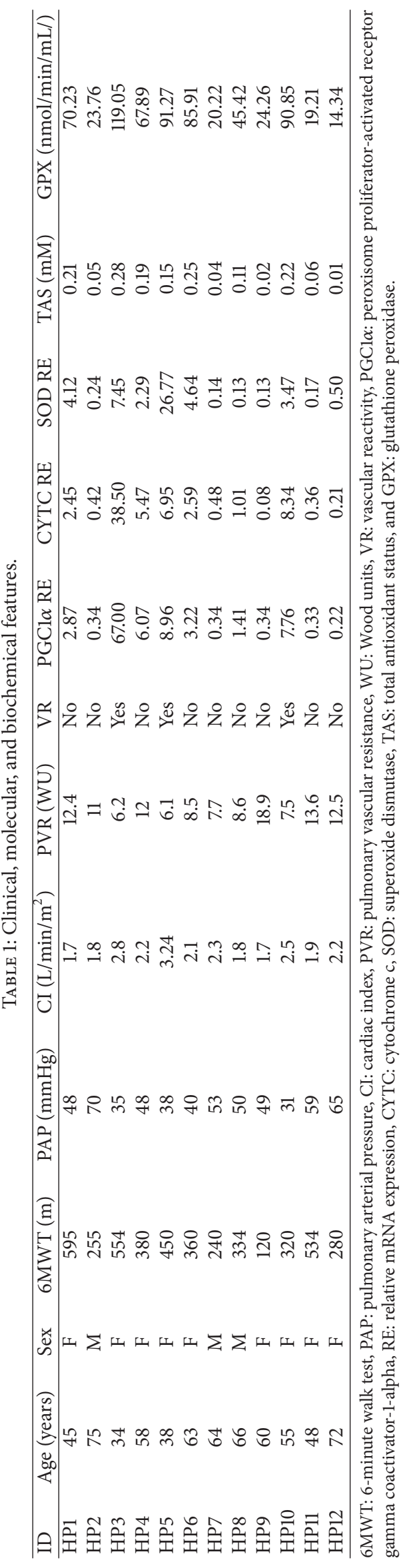


using a magnetic particle concentrator (MCP-1; Dynal) and washed four times with cold phosphate-buffered saline (PBS)/bovine serum albumin (BSA). Purified HSVPAEC retained on the CD-31-coated Dynabeads were separately resuspended in EGM-2 full growth medium supplemented with $10 \%$ FCS, $1 \%$ fungizone, and 2\% streptomycin/penicillin. The cells were grown in 24-well culture plates until $80 \%$ confluence. For miR23a inhibiting experiments, the lipofectamine ARNiMAX reagent (Invitrogen, USA) combined with the anti-miR23a MH10644 (Applied Biosystems, CA, USA) was used. Transfection experiments were done following the manufacturer instructions. Efficiency of transfection was evaluated using the GFP coding plasmid supplied by Altogen Biosystems (LA, USA) by fluorescence microscopy.

2.4. Real-Time RT-PCR. The relative expression levels of selected miRNAs were studied using real-time RT-PCR using standardized assays on demand (Applied Biosystems, CA, USA) and $10 \mathrm{ng}$ of the same extractions used for microarray analysis. The TaqMan microRNA reverse transcription kit was used for reverse transcription. For each miRNA included in the study we used specific primer supplied by manufacturer (Applied Biosystems, CA, USA). Reactions were performed in a Verity thermocycler (Applied Biosystems, CA, USA) following the manufacturer instructions. For RT-PCR the TaqMan Universal PCR Master Mix No AmpErase UNG (Applied Biosystems, CA, USA) was used with specific assay on demand for each miRNA analyzed. Expression data were obtained using a 7900HT real-time thermocycler (Applied Biosystems, CA, USA) and the relative expression was determined using the semicomparative $\Delta \Delta \mathrm{Ct}$ method, as reported in [19]. For normalization, the expression levels of miR374a, miR-374b, and let-7d were studied in the Affymetrix miRNA 2.0 arrays. These three miRNAs have been proposed as endogenous normalizers for serum microRNAs [20]. We found no significant changes in the expression of any of them and miR-374a was selected for normalization because it was the one that exhibited a lower variation across the data.

The relative expression of the PGC1 $\alpha, C Y C, S O D, N R F 2$, and $\mathrm{HO} 1$ genes was studied by real-time RT-PCR as described previously [6]. We used standardized assays on demand (Applied Biosystems, CA, USA) and $100 \mathrm{ng}$ of the same extractions used for total RNA microarray analysis. The cDNA was synthetized using the TaqMan reverse transcription reagents and random hexamers in a Verity thermocycler (Applied Biosystems, CA, USA). RT-PCR was performed in a 7900HT real-time thermocycler (Applied Biosystems, CA, USA) using the 2X Universal Master Mix (Applied Biosystems, CA, USA). The relative expression was determined using the semicomparative $\Delta \Delta \mathrm{Ct}$ method, as reported in [19]. For normalization, the human GAPDH was selected as housekeeping. The expression of this gene did not change between samples as it was corroborated in the microarrays performed.

2.5. Data Analysis. Data are presented as means \pm SEM of $n$ determinations. Statistical analysis of microarrays was done as described above. For the rest of the determinations, analysis of variance (ANOVA) followed by the Bonferroni test was applied using GraphPad Prism (GraphPad, San Diego, CA, USA). The $t$-test following false discovery rate (FDR) $P$ value correction was used to detect significant changes.

\section{Results}

3.1. miRNA Profile Changes in IPAH Patients. Circulating miRNA was extracted and analyzed using Affymetrix's microarrays as described above. In this study 12 wellcharacterized IPAH patients and 10 healthy volunteers were included. $t$-test following false discovery rate (FDR) $P$ value correction was used in order to detect significant changes. The results were filtered according to an absolute fold change $>2$ between the IPAH and healthy groups. We observed significant changes in 61 miRNAs; 36 were downregulated, while 25 were upregulated in the IPAH group compared to the healthy volunteers. Nonsupervised hierarchical clustering and principal component analysis (PCA) were used to classify the data. The results are shown in Figure 1; Table 2 lists all of the miRNA changes.

The data were validated using real-time RT-PCR. Due to the limitation of the sample, representative miRNAs were selected according to their biological significance or degree of change to be validated. We selected 15 miRNas corresponding to near the $25 \%$ of the data obtained. All miRNA analyzed by real-time RT-PCR was positively validated. The results are summarized in Table 3.

3.2. miR-23a Expression Is Correlated with Pulmonary Function Parameters. Multiple correlation analysis was used to explore the significance of the data. Pearson's correlation was used to compare the relative expression of relevant miRNAs (Table 3) and the value of different parameters indicative of the patients' pulmonary function, including patient age, the 6 min walk test (6MWT), MPAP, cardiac index (CI), and PVR. Of the miRNAs analyzed, significant correlations were found only for miR27a (6MWT), miR199a (6MWT and MPAP), miR744 (PVR), and miR23a (age, MPAP, CI, and PVR) (Table 4).

3.3. miR-23a Gene Targets Are Differentially Expressed in IPAH Patients. Based on the correlation analysis, we postulated that miR23a might serve as a biomarker of the pulmonary function of IPAH patients. Since miRNAs regulate gene expression, we explored whether miR23a gene targets were differentially expressed in IPAH patients and healthy volunteers. To this end, total RNA was extracted from peripheral blood of the same patients and subjected to global gene expression analysis using Affymetrix microarrays. Significant changes were filtered using ANOVA and FDR correction. The results were filtered according to an absolute fold change between the two groups $>2$. Genes whose expression is theoretically controlled by miR23a were identified by consulting the miRBase database (http://mirbase.org). Of the 132 genes with significantly changed expression between the IPAH patients and healthy volunteers, $22(\sim 17 \%)$ were potentially controlled at the expression level by miR23a. Interestingly, 
TABLE 2: Differentially expressed miRNAs in IPAH patients compared to healthy volunteers (HV).

\begin{tabular}{|c|c|c|c|}
\hline miRNA ID & $\begin{array}{l}\text { Average intensity } \\
(\mathrm{HV})\end{array}$ & $\begin{array}{c}\text { Average intensity } \\
\text { (IPAH) }\end{array}$ & Fold change \\
\hline miR-7-1 & 0.33 & 1.08 & 3.25 \\
\hline miR-204 & 0.78 & 0.17 & -4.67 \\
\hline miR-138-1 & 1.78 & 0.50 & -3.56 \\
\hline miR-520h & 0.78 & 0.17 & -4.67 \\
\hline miR-559 & 1.11 & 0.25 & -4.44 \\
\hline miR-593 & 0.67 & 0.17 & -4.00 \\
\hline miR-601 & 0.56 & 0.08 & -6.67 \\
\hline miR-616 & 0.89 & 0.17 & -5.33 \\
\hline miR-543 & 1.11 & 0.25 & -4.44 \\
\hline miR-1184 & 0.33 & 1.67 & 5.00 \\
\hline miR-1285 & 1.89 & 6.00 & 3.18 \\
\hline miR-1286 & 0.11 & 0.58 & 5.25 \\
\hline miR-3153 & 0.78 & 0.17 & -4.67 \\
\hline miR-3156 & 0.89 & 0.17 & -5.33 \\
\hline miR-4301 & 0.78 & 0.25 & -3.11 \\
\hline miR-4304 & 0.89 & 0.25 & -3.56 \\
\hline miR-4313 & 1.22 & 0.33 & -3.67 \\
\hline miR-1-2 & 0.11 & 0.92 & 8.25 \\
\hline miR-1259 & 0.67 & 0.17 & -4.00 \\
\hline miR-1263 & 0.22 & 0.83 & 3.75 \\
\hline miR-193a & 0.22 & 0.75 & 3.38 \\
\hline miR-195 & 0.22 & 0.75 & 3.38 \\
\hline miR-30c-2 & 0.67 & 0.08 & -8.00 \\
\hline miR-3120 & 0.89 & 0.17 & -5.33 \\
\hline miR-3145 & 0.67 & 0.17 & -4.00 \\
\hline miR-3184 & 0.33 & 1.08 & 3.25 \\
\hline miR-340 & 0.56 & 0.08 & -6.67 \\
\hline miR-4261 & 0.78 & 0.25 & -3.11 \\
\hline miR-524 & 0.56 & 0.08 & -6.67 \\
\hline miR-606 & 0.89 & 0.25 & -3.56 \\
\hline miR-634 & 0.56 & 0.08 & -6.67 \\
\hline miR-921 & 0.56 & 0.08 & -6.67 \\
\hline miR-99a & 0.67 & 0.08 & -8.00 \\
\hline miR-181d & 0.89 & 0.17 & -5.33 \\
\hline miR-1893 & 0.89 & 0.25 & -3.56 \\
\hline miR-1934 & 0.56 & 0.08 & -6.67 \\
\hline miR-1944 & 0.33 & 1.08 & 3.25 \\
\hline miR-1957 & 0.11 & 0.83 & 7.50 \\
\hline miR-1981 & 0.11 & 0.75 & 6.75 \\
\hline miR-20a & 0.25 & 0.79 & 3.15 \\
\hline miR-145 & 0.12 & 0.39 & 3.21 \\
\hline miR-27a & 0.17 & 0.53 & 3.12 \\
\hline miR-328 & 0.89 & 0.28 & -3.15 \\
\hline miR-23a & 0.21 & 0.65 & 3.09 \\
\hline miR-2145-2 & 0.22 & 0.75 & 3.38 \\
\hline miR-23b & 0.33 & 1.41 & 4.27 \\
\hline miR-291a & 0.56 & 0.08 & -6.67 \\
\hline
\end{tabular}

TABLE 2: Continued.

\begin{tabular}{lccc}
\hline miRNA ID & $\begin{array}{c}\text { Average intensity } \\
\text { (HV) }\end{array}$ & $\begin{array}{c}\text { Average intensity } \\
\text { (IPAH) }\end{array}$ & Fold change \\
\hline miR-191 & 0.11 & 0.58 & 5.25 \\
miR-327 & 0.11 & 0.58 & 5.25 \\
miR-423 & 0.22 & 0.92 & 4.13 \\
miR-465b-2 & 0.22 & 0.75 & 3.38 \\
miR-719 & 0.89 & 0.25 & -3.56 \\
miR-130 & 0.11 & 0.83 & 7.50 \\
miR-124-1 & 0.11 & 0.58 & 5.25 \\
miR-184 & 0.78 & 0.25 & -3.11 \\
miR-199a & 0.67 & 0.08 & -8.00 \\
miR-30e & 0.67 & 0.17 & -4.00 \\
miR-330 & 0.67 & 0.08 & -8.00 \\
miR-362 & 0.78 & 0.25 & -3.11 \\
miR-513 & 0.56 & 0.08 & -6.67 \\
miR-666 & 0.11 & 0.67 & 6.00 \\
\hline
\end{tabular}

TABLE 3: RT-PCR validation of differentially expressed miRNAs in IPAH patients compared to healthy volunteers (HV).

\begin{tabular}{lcc}
\hline miRNA ID & Microarray fold change & RT-PCR fold change \\
\hline miR-1-2 & 8.25 & 12.54 \\
miR-30c-2 & -8.00 & -5.46 \\
miR-99a & -8.00 & -7.25 \\
miR-1957 & 7.50 & 10.89 \\
miR-20a & 3.15 & 2.25 \\
miR-145 & 3.21 & 3.59 \\
miR-27a & 3.12 & 6.53 \\
miR-328 & -3.15 & -1.98 \\
miR-23a & 3.09 & 5.59 \\
miR-23b & 4.27 & 6.37 \\
miR-191 & 5.25 & 10.27 \\
miR-130 & 7.50 & 8.59 \\
miR-199a & -8.00 & -12.59 \\
miR-330 & -8.00 & -5.64 \\
miR-204 & -4.67 & -3.89 \\
\hline
\end{tabular}

one of them was $P G C 1 \alpha$, a gene whose expression was recently proposed as a potential biomarker in IPAH patients [6]. The results are shown in Figure 2 and Table 5.

3.4. miR-23a Expression Is Correlated with PGC1 $\alpha$ mRNA Levels. Finally, we examined the relationship between miR23a and PGCl $\alpha$ expression levels using linear regression analysis. We found a significantly negative correlation between the expression of both $(P=0.0001$, Pearson $r=-0.7985)$.

3.4.1. miR23a Inhibition Results in an Elevation of PGC1 $\alpha$, CYC, SOD, NRF2, and HO1 mRNA Expression Levels. To evaluate the possibility of a direct regulation of PGCl $\alpha$ by miR23a siRNA analysis experiments were carried out. HSVPAEC were cultured and miR23a expression was inhibited as described in the Section 2 for up to 72 hours. Every 24 hours 
TABLE 4: Multiple correlation analysis of pulmonary function parameters and miRNA expression.

\begin{tabular}{|c|c|c|c|c|c|c|c|c|c|c|}
\hline & \multicolumn{2}{|c|}{ 6MWT (m) } & \multicolumn{2}{|c|}{ Age (years) } & \multicolumn{2}{|c|}{ PAP (mmHg) } & \multicolumn{2}{|c|}{$\mathrm{CI}\left(\mathrm{L} / \mathrm{min} / \mathrm{m}^{2}\right)$} & \multicolumn{2}{|c|}{$\operatorname{PVR}\left(\mathrm{dyn} / \mathrm{sec} / \mathrm{cm}^{2}\right)$} \\
\hline & $P$ value & Pearson $r$ & $P$ value & Pearson $r$ & $P$ value & Pearson $r$ & $P$ value & Pearson $r$ & $P$ value & Pearson $r$ \\
\hline $\operatorname{miR} 27 a$ & 0.0082 & 0.452 & \multicolumn{2}{|c|}{ NS } & \multicolumn{2}{|c|}{ NS } & \multicolumn{2}{|c|}{ NS } & \multicolumn{2}{|c|}{ NS } \\
\hline miR23a & & NS & 0.0187 & -0.44 & 0.0001 & -0.618 & 0.0055 & 0.607 & 0,0426 & 0.795 \\
\hline miR199a & 0.0459 & 0.651 & \multicolumn{2}{|c|}{ NS } & 0.0474 & 0.753 & \multicolumn{2}{|c|}{ NS } & \multicolumn{2}{|c|}{ NS } \\
\hline miR744 & \multicolumn{2}{|r|}{ NS } & & $S$ & \multicolumn{2}{|c|}{ NS } & \multicolumn{2}{|c|}{ NS } & 0.0124 & 0.851 \\
\hline
\end{tabular}

6MWT: 6-minute walk test, PAP: pulmonary arterial pressure, CI: cardiac index, PVR: pulmonary vascular resistance, and NS: nonsignificant.

TABLE 5: Differentially expressed RNAs in IPAH patients compared to healthy volunteers (HV).

\begin{tabular}{lccc}
\hline Gene symbol & $\begin{array}{c}\text { Average intensity } \\
(\mathrm{HV})\end{array}$ & $\begin{array}{c}\text { Average intensity } \\
(\text { IPAH })\end{array}$ & Fold change \\
\hline CAB39 & 2.39 & 0.72 & -3.32 \\
SLC4A4 & 0.88 & 2.15 & 2.45 \\
PDE7A & 2.59 & 1.09 & -2.38 \\
PPARGC1A & 1.36 & 3.19 & 2.35 \\
DEPDC1 & 0.96 & 3.13 & 3.26 \\
TMPO & 1.91 & 0.45 & -4.21 \\
PCDH18 & 0.98 & 2.09 & 2.13 \\
ZNF208 & 1.16 & 2.30 & 1.98 \\
IL6R & 2.15 & 4.49 & 2.09 \\
CEP85L & 2.51 & 1.08 & -2.31 \\
CENPN & 1.01 & 6.31 & 6.25 \\
HPGD & 1.92 & 0.84 & -2.30 \\
NAA15 & 2.36 & 0.71 & -3.31 \\
ST18 & 1.13 & 2.98 & 2.64 \\
NKAIN2 & 1.02 & 4.32 & 4.23 \\
SEC23A & 2.54 & 0.78 & -3.25 \\
NOX4 & 1.04 & 4.25 & 4.09 \\
ODF2L & 1.66 & 0.18 & -9.47 \\
MBNL1 & 2.77 & 1.16 & -2.39 \\
TNKS2 & 2.37 & 0.66 & -3.57 \\
ZNF423 & 1.12 & 2.78 & 2.48 \\
ZNF117 & 1.21 & 3.04 & 2.51 \\
\hline
\end{tabular}

total RNA was extracted and relative expression of PGC1 $\alpha$ as well as its well-known related genes CYC, SOD, and NRF2 were analyzed. The results obtained are shown in Figure 3. The relative expression levels of PGCl $\alpha$ were increased in anti-miR23a transfected cells compared to mock cultures (Figure 3(a)). This change was in parallel with an increase in the relative expression of $C Y C, S O D$, and NRF2 (Figure 3(b)).

\section{Discussion}

This study compared the expression profiles of circulating miRNAs in 12 well-characterized patients and ten healthy volunteers. We found significant changes in 61 miRNAs, as supported by nonsupervised hierarchical clustering and PCA analysis. Different studies have proposed that miRNAs are involved in the regulation of the pathophysiological events leading to the development of PAH.
In this regard, we highlight the relevance of miR204, which is aberrantly expressed in human PAH smooth muscle cells. Its expression correlates with the pulmonary function of PAH patients and its inhibition is related to an increase in smooth muscle cell proliferation and resistance to apoptosis $[21,22]$. It was also associated with the protective action of exosomes in a murine model of hypoxic PAH [23]. The miR204 levels were significantly lower in the IPAH subjects compared to the healthy volunteers, supporting the importance of miR204 in the pathogenesis of IPAH [24].

Another important miRNA associated with PH is miR145. Its expression is augmented in patients with heritable PAH and IPAH and BMPR2 mutations increased its expression in mice and PAH patients [25]. We found that miR145 was upregulated in the blood of IPAH patients, supporting its important role in the pathophysiology of PAH. In addition, we found significant changes in miR27a, which is overexpressed in patients with hereditary $\mathrm{PAH}$ and is associated with the control of BMPR2-mediated cell proliferation [26], and in miR328, which induces autoapoptosis in smooth muscle cells, inhibiting IGFR1, and acts as a protective agent in PAH [27].

In addition, we observed significant changes in miRNAs related to other pathologies, but not to $\mathrm{PAH}$, including miR1-2, miR-330, miR-199a, and miR-744. miR-1-2 is involved in the regulation of cardiogenesis, promoting the differentiation of cardiac stem cells into cardiomyocytes; it is also associated with the induction of the antioxidant response of skeletal muscle [28-30]. miR-330 controls the expression of different genes in renal smooth muscle [31]. miR-199a plays an important role in the regulation of HIF1A in cardiomyocytes in hypoxic environments [32]. miR-744 is overexpressed in several cancers, including that of the head and neck [33]. All of these factors are involved in the oxidative stress response, and most regulate the responses of different types of muscle cell, which is in concordance with some of the cellular events involved in $\mathrm{PAH}$.

One of the most interesting miRNAs identified in this study was miR-23a. The overexpression of miR-23a induces cardiac hypertrophy in mice [34]. We found that its expression was increased in IPAH patients compared to the healthy volunteers. There were good correlations with age, MPAP, CI, and PVR, which supports the use of this miRNA as a biomarker of the pulmonary function of IPAH patients. Interestingly, $17 \%$ of the genes whose expression levels were changed significantly in IPAH patients compared with the healthy volunteers are theoretically regulated by miR23a. 


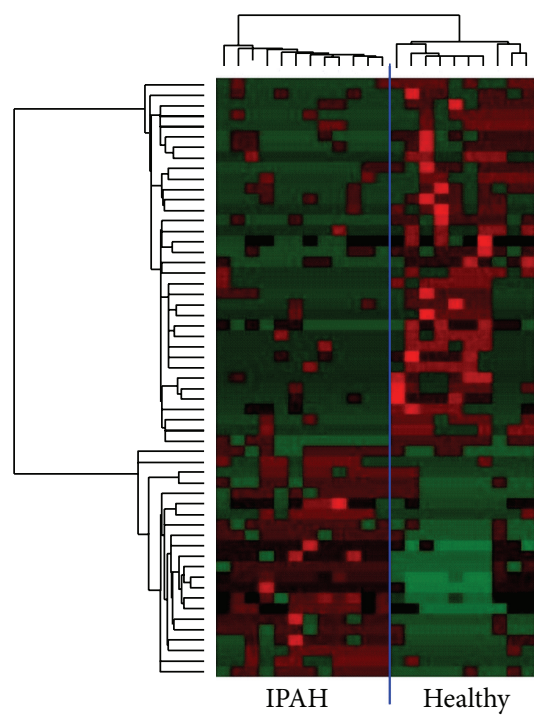

(a)

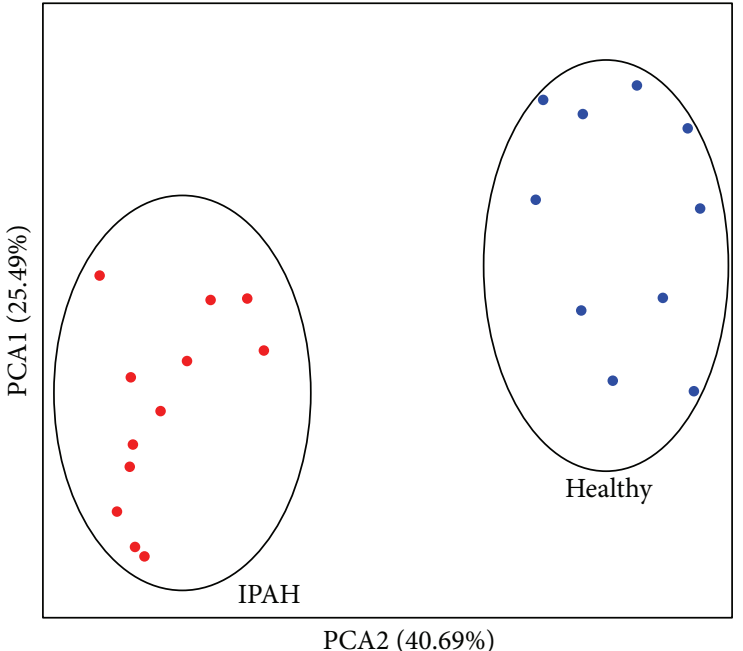

(b)

FIGURE 1: Circulating miRNAs differentially expressed in IPAH patients compared with healthy volunteers. Circulating miRNAs were extracted from blood from 12 IPAH patients and 10 healthy volunteers and analyzed using Affymetrix miRNA arrays. The $t$-test following the false discovery rate (FDR) $P$ value correction was used to detect significant changes. Hierarchical clustering (a) and principal component analysis (PCA, (b)) were used to analyze the data obtained.

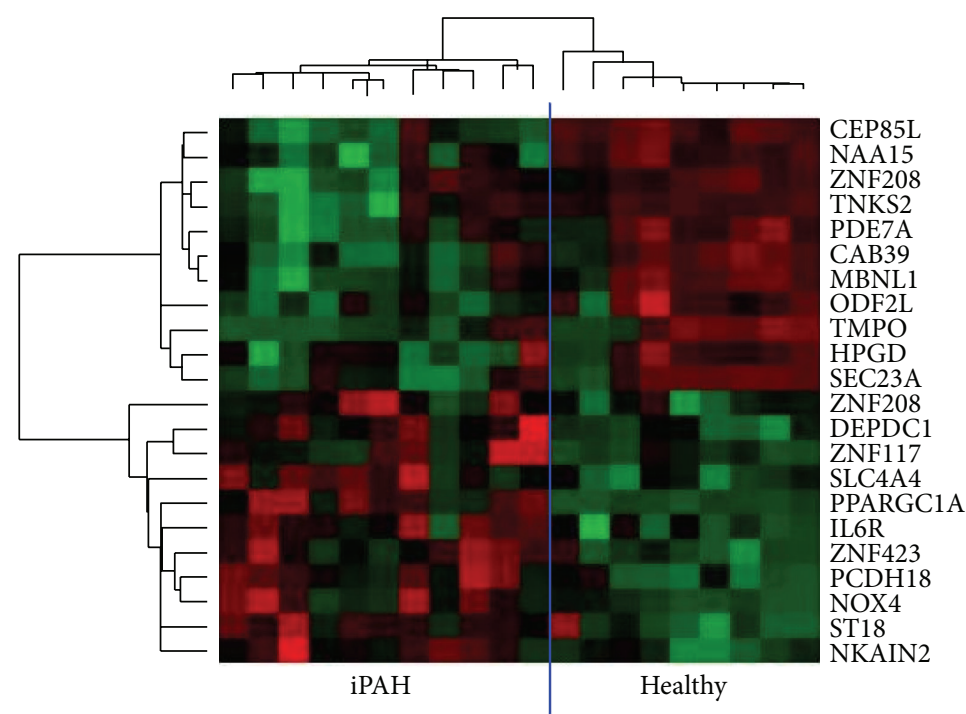

(a)

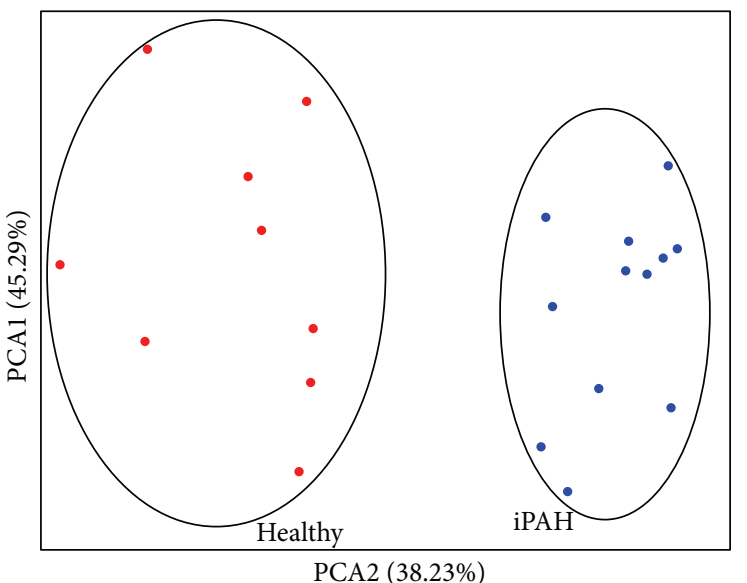

(b)

FIGURE 2: Circulating RNAs differentially expressed in IPAH patients compared with healthy volunteers. Circulating total RNAs were extracted from blood from 12 IPAH patients and 10 healthy volunteers and analyzed using Affymetrix miRNA arrays. The $t$-test following the false discovery rate (FDR) $P$ value correction was used to detect significant changes. Hierarchical clustering (a) and principal component analysis (PCA, (b)) were used to analyze the data obtained.

Among these, we highlight PGC1 $\alpha$. This protein acts as a coactivator of PPAR $\gamma$ and it was recently proposed to be an important regulator of the antioxidant response of IPAH patients and a biomarker of the pulmonary status of IPAH patients [6]. We found a significant negative correlation between miR23a and PGC1 $\alpha$ expression, which concurs with recent evidence that miR23a inhibits PGC1 $\alpha$ expression directly in hepatocellular carcinoma via the IL6Stat3 signaling pathway [35]. We found upregulated PGC1 $\alpha$ expression in the IPAH patients compared to the healthy volunteers. This increase in PGC1 $\alpha$ expression is probably caused by a response to the hypoxia to which these patients are subjected, although this response is insufficient to ameliorate the effects of hypoxia. One of the factors that could be 


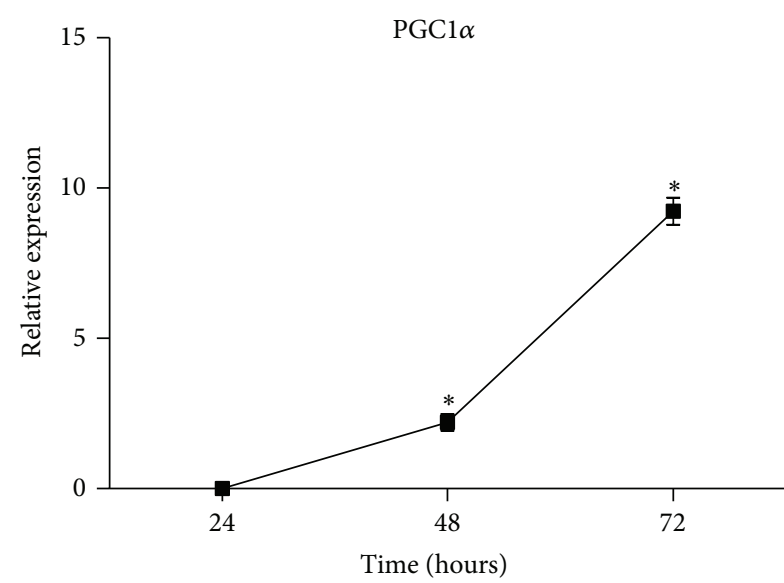

(a)

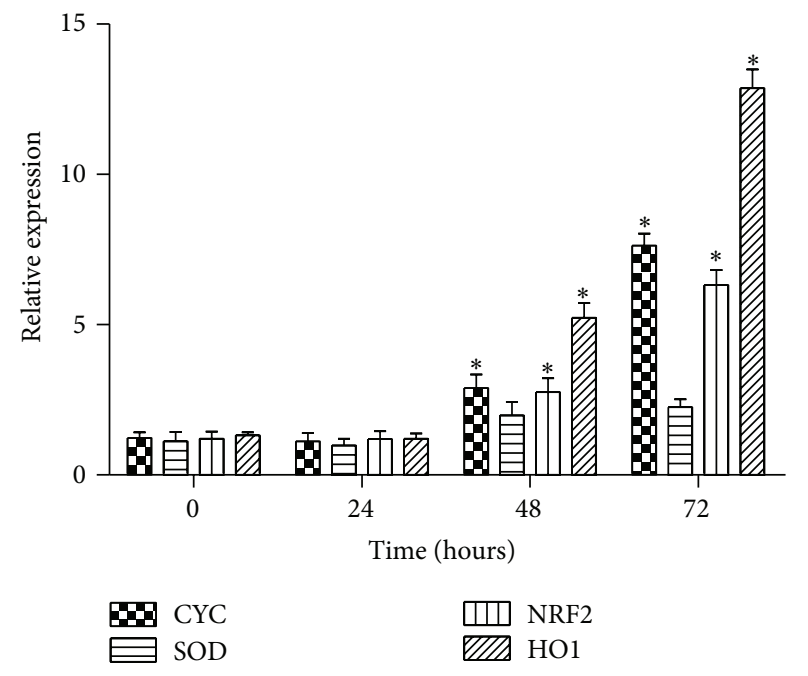

(b)

FIGURE 3: miR23a inhibition results in an increase in the relative expression levels of PGC1 $\alpha$. Human small vascular pulmonary artery endothelial cells (HSVPAEC) were cultured and transfected with the anti-miR23a MH10644 for up to 72 hours. Every 24 hours total RNA was extracted and analyzed by real-time RT-PCR for PGC1 $\alpha$ (a) or for CYC, SOD, NRF2, and HO1 (b). Three independent experiments were included in each experimental group. Results are represented as mean \pm SEM. ${ }^{*} P<0.05$ compared to mock transfected cells.

involved in this failure is the abnormal expression of miR23a, which could partially repress the induction of PGCl $\alpha$. To explore this, siRNA experiments have been carried out in cultured human small vasculature pulmonary endothelial cells. Our result indicates that after silencing of miR23a there is a significant increase of the mRNA levels of PPGClalpha, as well as in the well-known related genes CYC, SOD, NRF2, and $H O 1$, which enforces the idea of this relation and is in line with results reported by other authors [36].

Another gene whose expression is controlled by miR23a and was upregulated in the IPAH group is IL6R, which is implicated in the IL6-Stat3 signaling pathway. Monoclonal antibodies that blocked this receptor inhibited breast tumor proliferation and reduced circulating cancer cells, supporting the importance of this pathway in the abnormal proliferation of cells that affect the pulmonary vasculature of IPAH patients [37].

One limitation of this study involves the different treatment supplied to the patients analyzed including different combinations of bosentan, treprostinil, nifedipine, and iloprost. Probably several of the circulating miRNAs involved in the pathogenesis of IPAH are being masked due to the effect of the treatment. More studies including the novo diagnosed patients before and after treatment are needed in order to identify these miRNAs as well as the specific effect of each of these drugs modulating the expression of these masked miRNAs. Data provided here support the usefulness of the analysis of circulating miRNAs as potential biomarkers of the progression of IPAH as well as the possible relevance of miR23a which may be involved in the control of several genes related to the IPAH including PGCl $\alpha$. Nevertheless, more studies are needed to analyze specificity and sensitivity of this molecular tool to be used as biomarker of the IPAH.

\section{Conflict of Interests}

The authors declare that there is no conflict of interests regarding the publication of this paper.

\section{Acknowledgment}

This work was supported by Grants PI10/02294 (Manuel Mata Roig), SAF2011-26443 (Julio Cortijo Gimeno), and CIBERES (CB06/06/0027) from the Health Institute "Carlos III" of the Spanish government.

\section{References}

[1] S. Rich, D. R. Dantzker, S. M. Ayres et al., "Primary pulmonary hypertension. A national prospective study," Annals of Internal Medicine, vol. 107, no. 2, pp. 216-223, 1987.

[2] V. V. McLaughlin, S. L. Archer, D. B. Badesch et al., "ACCF/AHA. ACCF/AHA 2009 expert consensus document on pulmonary hypertension: a report of the American College of Cardiology Foundation Task Force on Expert Consensus Documents and the American Heart Association: developed in collaboration with the American College of Chest Physicians, American Thoracic Society, and the Pulmonary Hypertension Association," Circulation, vol. 119, no. 16, pp. 2250-2294, 2009.

[3] N. Galiè, M. M. Hoeper, M. Humbert et al., "ESC Committee for Practice Guidelines (CPG). Guidelines for the diagnosis and treatment of pulmonary hypertension: the Task Force for the Diagnosis and Treatment of Pulmonary Hypertension of the European Society of Cardiology (ESC) and the European Respiratory Society (ERS), endorsed by the International Society of Heart and Lung Transplantation (ISHLT)," European Heart Journal, vol. 30, no. 20, pp. 2493-2537, 2009. 
[4] M. Sano, T. Minamino, H. Toko et al., "p53-induced inhibition of Hif-1 causes cardiac dysfunction during pressure overload," Nature, vol. 446, no. 7134, pp. 444-448, 2007.

[5] N. F. Voelkel, R. A. Quaife, L. A. Leinwand et al., "Right ventricular function and failure: report of a National Heart, Lung, and Blood Institute working group on cellular and molecular mechanisms of right heart failure," Circulation, vol. 114, no. 17, pp. 1883-1891, 2006.

[6] M. Mata, I. Sarrion, L. Milian et al., "PGC-1 $\alpha$ induction in pulmonary arterial hypertension," Oxidative Medicine and Cellular Longevity, vol. 2012, Article ID 236572, 8 pages, 2012.

[7] Z. Wu, P. Puigserver, U. Andersson et al., "Mechanisms controlling mitochondrial biogenesis and respiration through the thermogenic coactivator PGC-1," Cell, vol. 98, no. 1, pp. 115-124, 1999.

[8] D. P. Bartel, "MicroRNAs: genomics, biogenesis, mechanism, and function," Cell, vol. 116, no. 2, pp. 281-297, 2004.

[9] D. S. Schwarz, G. Hutvágner, T. Du, Z. Xu, N. Aronin, and P. D. Zamore, "Asymmetry in the assembly of the RNAi enzyme complex," Cell, vol. 115, no. 2, pp. 199-208, 2003.

[10] V. Di Stefano, G. Zaccagnini, M. C. Capogrossi, and F. Martelli, "microRNAs as peripheral blood biomarkers of cardiovascular disease," Vascular Pharmacology, vol. 55, no. 4, pp. 111-118, 2011.

[11] G.-K. Wang, J.-Q. Zhu, J.-T. Zhang et al., "Circulating microRNA: a novel potential biomarker for early diagnosis of acute myocardial infarction in humans," European Heart Journal, vol. 31, no. 6, pp. 659-666, 2010.

[12] O. Gidlöf, P. Andersson, J. van der Pals, M. Götberg, and D. Erlinge, "Cardiospecific microRNA plasma levels correlate with troponin and cardiac function in patients with ST elevation myocardial infarction, are selectively dependent on renal elimination, and can be detected in urine samples," Cardiology, vol. 118, no. 4, pp. 217-226, 2011.

[13] X. Ji, R. Takahashi, Y. Hiura, G. Hirokawa, Y. Fukushima, and N. Iwai, "Plasma miR-208 as a biomarker of myocardial injury," Clinical Chemistry, vol. 55, no. 11, pp. 1944-1949, 2009.

[14] A. Lee, D. McLean, J. Choi, H. Kang, W. Chang, and J. Kim, "Therapeutic implications of microRNAs in pulmonary arterial hypertension," BMB Reports, vol. 47, no. 6, pp. 311-317, 2014.

[15] W. Y. Li, J. Jin, J. Chen, Y. Guo, J. Tang, and S. Tan, "Circulating microRNAs as potential non-invasive biomarkers for the early detection of hypertension-related stroke," Journal of Human Hypertension, vol. 28, no. 5, pp. 288-291, 2014.

[16] C. Li and W. H. Wong, "Model-based analysis of oligonucleotide arrays: expression index computation and outlier detection," Proceedings of the National Academy of Sciences of the United States of America, vol. 98, no. 1, pp. 31-36, 2001.

[17] M. Mata, F. Pallardo, E. J. Morcillo, and J. Cortijo, "Piclamilast inhibits the pro-apoptotic and anti-proliferative responses of A549 cells exposed to $\mathrm{H}_{2} \mathrm{O}_{2}$ via mechanisms involving AP-1 activation," Free Radical Research, vol. 46, no. 5, pp. 690-699, 2012.

[18] J. L. Ortiz, J. Milara, G. Juan et al., "Direct effect of cigarette smoke on human pulmonary artery tension," Pulmonary Pharmacology and Therapeutics, vol. 23, no. 3, pp. 222-228, 2010.

[19] M. Mata, B. Sarriá, A. Buenestado, J. Cortijo, M. Cerdá, and E. J. Morcillo, "Phosphodiesterase 4 inhibition decreases MUC5AC expression induced by epidermal growth factor in human airway epithelial cells," Thorax, vol. 60, no. 2, pp. 144-152, 2005.

[20] R. Qi, M. Weiland, X. H. Gao, L. Zhou, and Q.-S. Mi, "Identification of endogenous normalizers for serum MicroRNAs by microarray profiling: U6 small nuclear RNA is not a reliable normalizer," Hepatology, vol. 55, no. 5, pp. 1640-1642, 2012.

[21] A. Courboulin, R. Paulin, N. J. Giguère et al., "Role for miR204 in human pulmonary arterial hypertension," Journal of Experimental Medicine, vol. 208, no. 3, pp. 535-548, 2011.

[22] C. L. Bockmeyer, L. Maegel, S. Janciauskiene et al., "Plexiform vasculopathy of severe pulmonary arterial hypertension and microRNA expression," Journal of Heart and Lung Transplantation, vol. 31, no. 7, pp. 764-772, 2012.

[23] C. Lee, S. A. Mitsialis, M. Aslam et al., "Exosomes mediate the cytoprotective action of mesenchymal stromal cells on hypoxiainduced pulmonary hypertension," Circulation, vol. 126, no. 22, pp. 2601-2611, 2012.

[24] C. Wei, H. Henderson, C. Spradley et al., "Circulating miRNAs as potential marker for pulmonary hypertension," PLoS ONE, vol. 8, no. 5, Article ID e64396, 2013.

[25] P. Caruso, Y. Dempsie, H. C. Stevens et al., "A role for miR-145 in pulmonary arterial hypertension: evidence from mouse models and patient samples," Circulation Research, vol. 111, no. 3, pp. 290-300, 2012.

[26] K. M. Drake, D. Zygmunt, L. Mavrakis et al., "Altered microRNA processing in heritable pulmonary arterial hypertension: an important role for Smad-8," The American Journal of Respiratory and Critical Care Medicine, vol. 184, no. 12, pp. 1400-1408, 2011.

[27] L. Guo, Z. Qiu, L. Wei et al., "The MicroRNA-328 regulates hypoxic pulmonary hypertension by targeting at insulin growth factor 1 Receptor and L-type calcium channel- $\alpha 1 C$," Hypertension, vol. 59, no. 5, pp. 1006-1013, 2012.

[28] Y. Mishima, C. Stahlhut, and A. J. Giraldez, "miR-1-2 gets to the heart of the matter," Cell, vol. 129, no. 2, pp. 247-249, 2007.

[29] K. R. Cordes, D. Srivastava, and K. N. Ivey, "MicroRNAs in cardiac development," Pediatric Cardiology, vol. 31, no. 3, pp. 349-356, 2010.

[30] A. Safdar, A. Abadi, M. Akhtar, B. P. Hettinga, and M. A. Tarnopolsky, "miRNA in the regulation of skeletal muscle adaptation to acute endurance exercise in $\mathrm{C} 57 \mathrm{Bl} / 6 \mathrm{~J}$ male mice," PLoS ONE, vol. 4, no. 5, Article ID e5610, 2009.

[31] S. Medrano, M. C. Monteagudo, M. L. S. Sequeira-Lopez, E. S. Pentz, and R. Ariel Gomez, "Two microRNAs, miR330 and miR-125b-5p, mark the juxtaglomerular cell and balance its smooth muscle phenotype," The American Journal of Physiology_Renal Physiology, vol. 302, no. 1, pp. 29-37, 2012.

[32] S. Rane, M. He, D. Sayed et al., "Downregulation of MiR199a derepresses hypoxia-inducible factor- $1 \alpha$ and sirtuin 1 and recapitulates hypoxia preconditioning in cardiac myocytes," Circulation Research, vol. 104, no. 7, pp. 879-886, 2009.

[33] V. Huang, R. F. Place, V. Portnoy et al., "Upregulation of cyclin B1 by miRNA and its implications in cancer," Nucleic Acids Research, vol. 40, no. 4, pp. 1695-1707, 2012.

[34] E. van Rooij, L. B. Sutherland, N. Liu et al., "A signature pattern of stress-responsive microRNAs that can evoke cardiac hypertrophy and heart failure," Proceedings of the National Academy of Sciences of the United States of America, vol. 103, no. 48, pp. 18255-18260, 2006.

[35] B. Wang, S.-H. Hsu, W. Frankel, K. Ghoshal, and S. T. Jacob, "Stat3-mediated activation of microRNA-23a suppresses gluconeogenesis in hepatocellular carcinoma by down-regulating Glucose-6-phosphatase and peroxisome proliferator-activated receptor gamma, coactivator 1 alpha," Hepatology, vol. 56, no. 1, pp. 186-197, 2012. 
[36] B. Wang, S. H. Hsu, W. Frankel, K. Ghoshal, and S. T. Jacob, "Stat3-mediated activation of microRNA-23a suppresses gluconeogenesis in hepatocellular carcinoma by down-regulating Glucose-6-phosphatase and peroxisome proliferator-activated receptor gamma, coactivator 1 alpha," Hepatology, vol. 56, no. 1, pp. 186-197, 2012.

[37] G. Kim, M. Ouzounova, A. A. Quraishi et al., "SOCS3-mediated regulation of inflammatory cytokines in PTEN and p53 inactivated triple negative breast cancer model," Oncogene, vol. 34, pp. 671-680, 2015. 


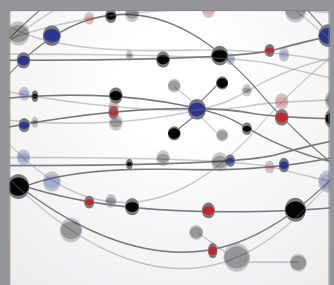

The Scientific World Journal
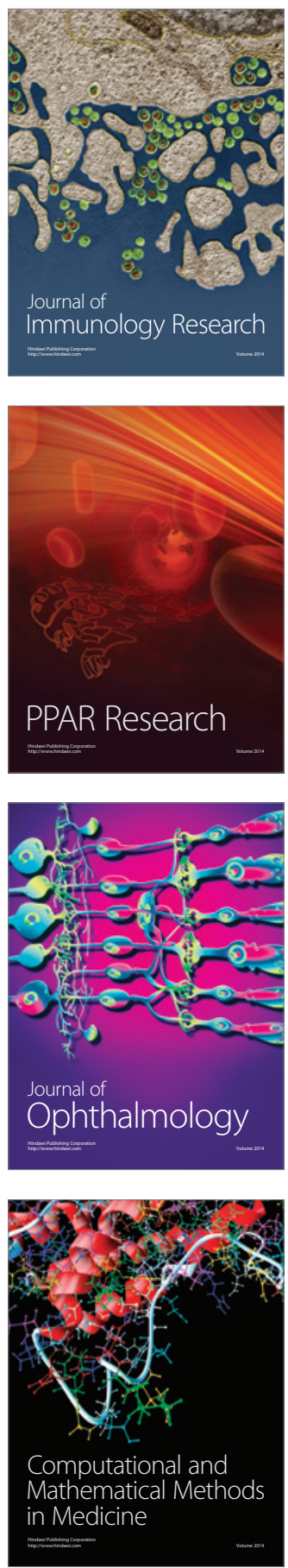

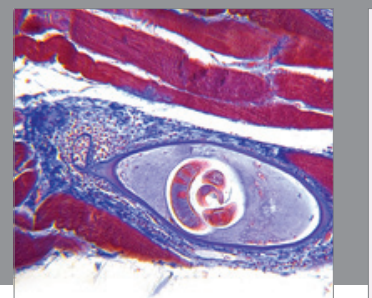

Gastroenterology

Research and Practice
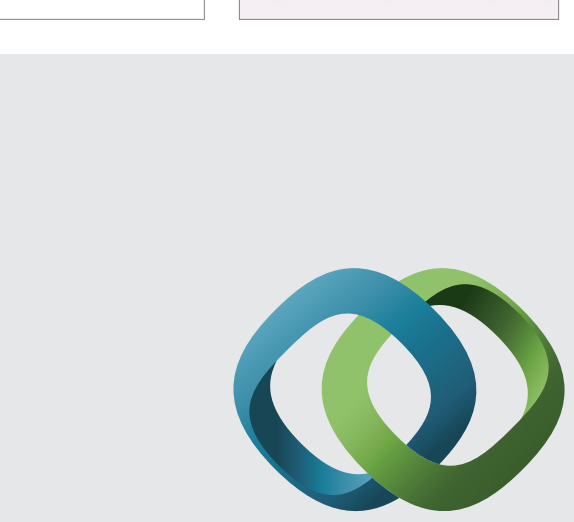

\section{Hindawi}

Submit your manuscripts at

http://www.hindawi.com
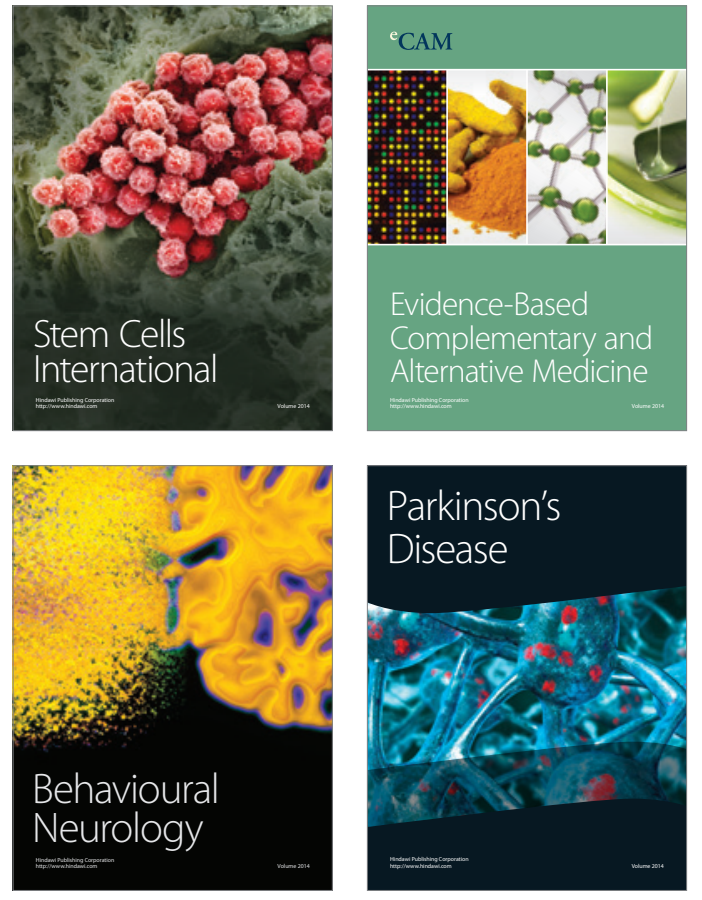
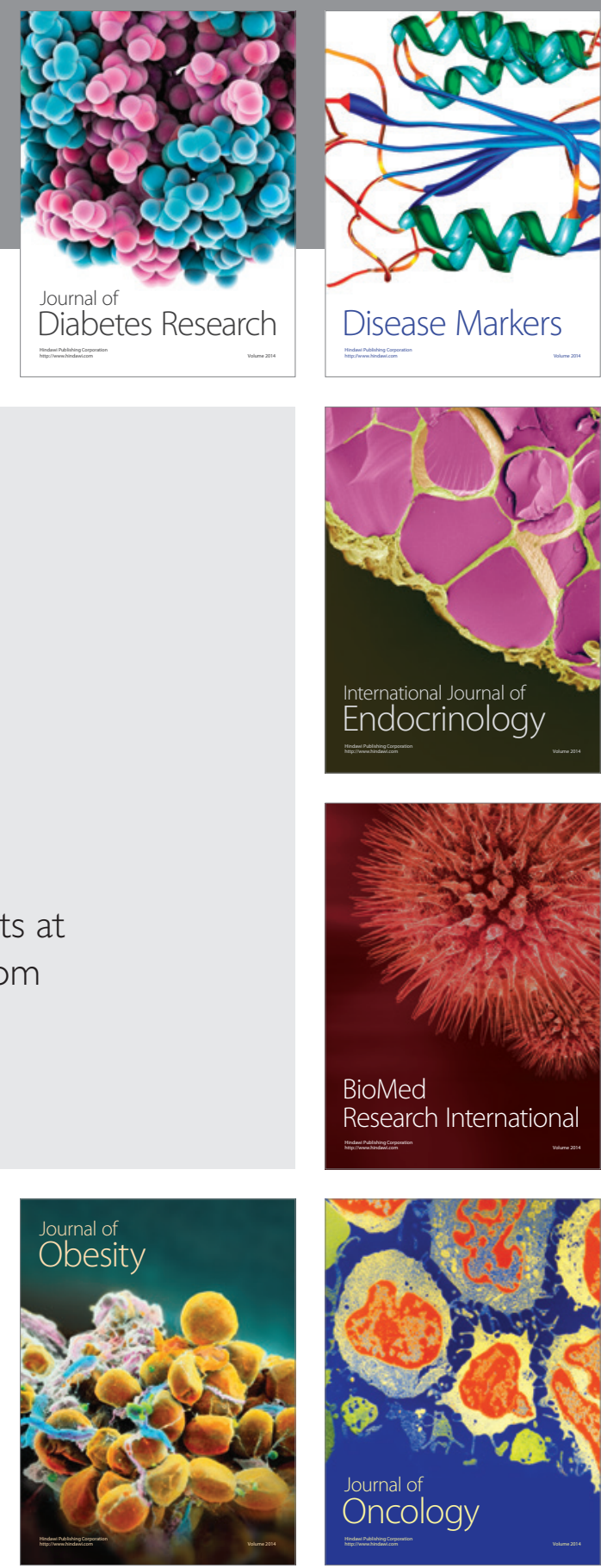

Disease Markers
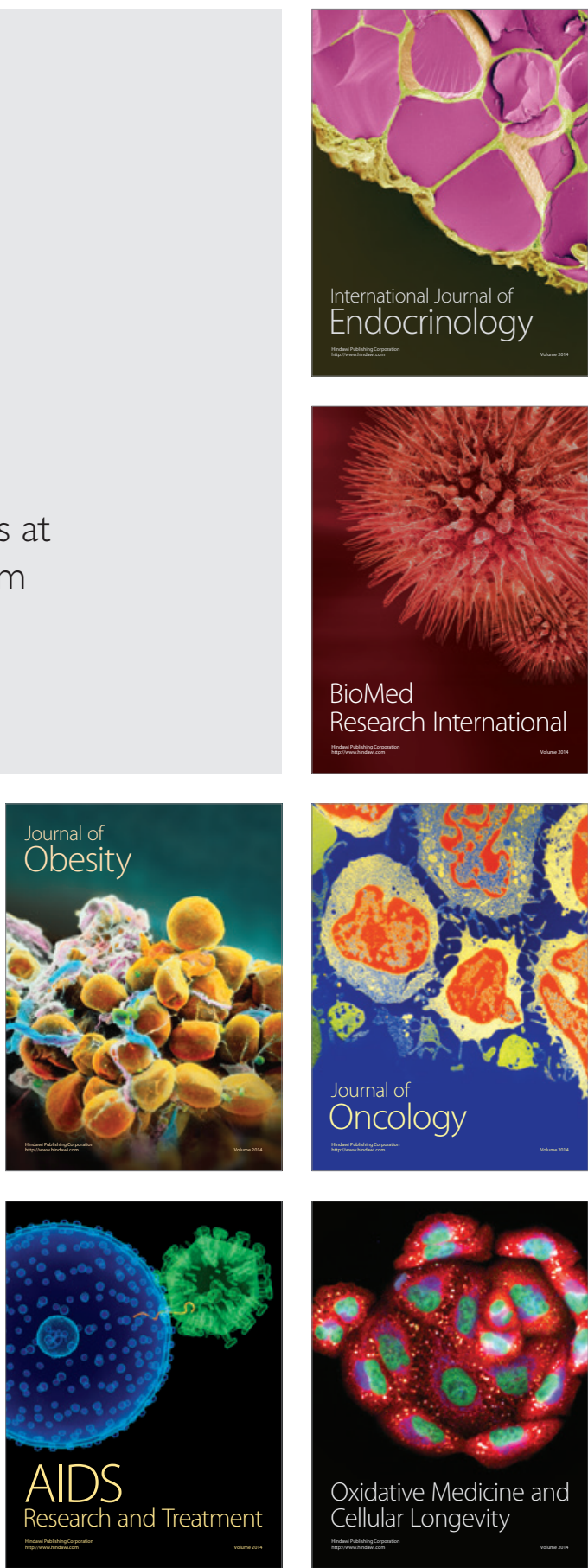